\title{
Design and Performance Analysis of Sensor Proxy-AAA Authentication Scheme Based on Fast Handover and Forwarding Mode for IP-based Internet of Things
}

\author{
Chulhee Cho $^{1}$, Byung-Hun Song ${ }^{2}$, Jongpil Jeong ${ }^{3}$, Tai-Myoung Chung ${ }^{1, *}$ \\ ${ }^{1}$ College of Information and Communica tions Engineering, Sungkyunkw an University, Suwon, Kyung gi-do, \\ 440-745, Republic of Korea \\ ${ }^{2}$ IoT Convergence Research Center, Korea Electronics Technology Institute (KETI), Seongnam, Republic of Korea \\ ${ }^{3}$ Department of Human ICT Convergence, Sungkyunkwan University, Suwon, Kyung gi-do 440-745, Republic of \\ Korea
}

\section{Abstract}

Recen tly interest in Internet of Things(I oT) is increasing, and a variety of the security technol ogies that are suitable for Internet of Things has being studied. In order to main tain the trustw orthy connectivity and the accessibility of distributed IoT, it is importan t to establish secure links for end-to-end comm unica tion with proper authen tication. AAA technol ogy is curren tly the best way of resolving dela y issue when introducing authen tica tion process of mobile switching. However, there are still a number of issues among which the dela $y$ time issue from authen tication and authoriza tion grea tly inf uences the process. AAA applica tion in mobile IP environmen $t$ cannot $f$ uently support continuous and fast handov er in both intra-domain and inter-domain. Mobile IPv6 (MIPv6) is a host-based protocol supporting global mobility. On the other hand, Proxy Mobile IPv6 (PMIPv6) is a netw ork-based protocol supporting localized mobility. This paper, the additional cost from combina tion of PMIPv6, authen tication, authoriza tion and accoun ting (AAA) and the way of red ucing extended delay time will be explained. First, a new authen tication scheme (Proxy-AAA) is proposed that supports forwarding mode and fast handov er mode betw een other local mobility anchors (LMAs). Second, configur cost analysis model based on Proxy-AAA. Based on theoretical analysis, it was confirme that the cost is affected by average arriv al rate and residence time.

Receiv ed on 26 November 2016; accepted on 13 July 2017; published on 13 September 2017

Keywords: Proxy-AAA, Forwarding, inter-domain handov er, re-using the session key

Copyright (C) 2017 Chulhee Cho et al., licensed to EAI. This is an open access article distributed under the terms of the Crea tive Commons Attribution license (http://creativecommons.org/licenses/by/3.0/), which permits unlimited use, distribution and reprod uction in any medium so long as the original work is proper ly cited.

doi:10.4108/eai.13-9-2017.153338

\section{Introduction}

Recen tly interest in Internet of Things(I oT) is increasing, and a variety of the security technol ogies that are

\footnotetext{
« This article is a revised and expanded version of a paper entitled "Design and Performance Analysis of Sensor Proxy-AAA Authen tication Scheme Based on Fast Handover and Forwarding Mode for IP-based Internet of Things", presen ted at 12th EAI Interna tional Conference on Heterog eneous Netw orking for Quality, Reliability, Security and Robustness, JULY 7-8, 2016 | SEOUL, SOUTH KOREA.

${ }^{*}$ Corresponding author: Tai-Myoung Chung, Ph.D., research fie d: distributed computing. Email: tmchung@ece.skku.ac.kr
}

suitable for Internet of Things has being studied. Especiall y sensor netw ork area of the device is an increased using and diversifie for a low specific tion devices beca use of char acteristic of the Internet of Things. Many entities sensor nodes may move around in a real world environmen $t$, thus making the IoT devices attached to them mobile. In order to main tain the trustw orthy connectivity and the accessibility of distributed IoT, it is importan t to establish secure links for end-toend comm unica tion with proper authen tication. In the internet of things environmen $t$, due to the open char acteristic of internet of things, the security issue related 
to authen tication of user accessing wireless netw ork is extremel y importan t. AAA technol ogy is curren tly the best way of resolving dela $y$ issue when introducing authen tica tion process of mobile switching $[1,2]$. How ever, despite long developmen $\mathrm{t}$ of AAA technol ogy, the mobility manag emen $t$ in wireless netw ork environmen $t$ has yet to be researched further. With the distribution of MIPv6 netw ork and developmen $t$ of new access technol ogies, the UDP-based Remote Authen tication Dial-In User Service (RADIUS) protocol can no longer satisfy requiremen ts. Diameter protocol, an improved version from RADIUS, provides extremel y improved functions in failure recovery, security and reliability [3]. How ever, the dela y from authen tication and authorization process greatly inf uences the process and AAA applica tion in mobile IP has a number of issues such as failing to support continuous and fast handover in both intra-domain and inter-domain [4-7]. Moreover, another mobility manag emen t protocol called PMIPv6 is in the limelight. PMIPv6 is an enhancemen t of MIPv6 and provides a netw ork-based localized mobility managemen $t$ with support for legacy mobile devices [8]. Due to its differen t char acteristics from MIPv6, PMIPv6 can be introduced along with MIPv6. For example, MIPv6 can be used for global mobility while PIMPv6 can be used in intra-domain mobility [9]. To address the shortcomings of the above mentioned schemes, this paper presen ts a Proxy-Authen tication Authoriza tion Accoun ting (Proxy-AAA) authen tica tion scheme. In this proposed technique, the AAA server will be implemented on Local Mobility Anchor (LCA) to implemen $t$ fast handov er authen tica tion and hier archical authentication as well as reduce intra-domain authen tication cost $[10,11]$. The perf ormance of Mobile IPv6 (MIPv6) and Proxy-AAA scheme to select the appropria te protocol was evaluated. Netw ork status and mobility parameters can be better selected according to the protocol. For the proposed Proxy-AAA, signaling overhead is alw ays less than with the traditional AAA method. Also, in cases where the Mobile Node (MN) moves farther away from the home domain, the proposed scheme is more efficien than the traditional AAA scheme $[12,13]$. We firs describe and compare basic MIPv6 and PMIPv6 and describe 6LoWPAN netw ork in section 2. In section 3 , we introduce our proposed Proxy-AAA and protocol selection scheme. In section 4 , the performance of the traditional AAA scheme and proposed Proxy-AAA scheme is compared. Section 5 concludes the paper with a summary of the key resul ts of this work.

\section{Related Work}

\subsection{Comparison of MIPv6 and PMIPv6}

MIPv6 supports host-based mobility to $\mathrm{MN}$ and reduces high mobility signaling overhead while $\mathrm{MN}$ implemen ts frequen $t$ handov er betw een subnets [14,

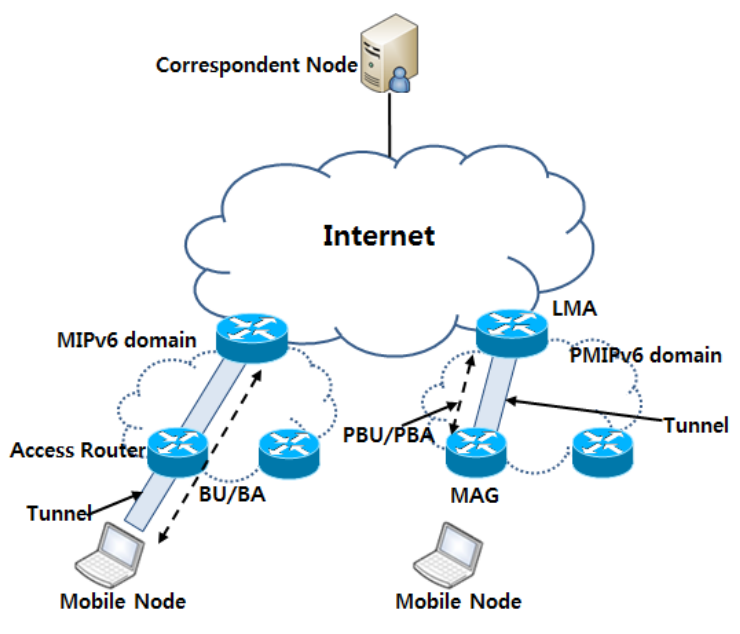

Figure 1. The architectures of MIPv6 and PMIPv6.

15]. PMIPv6 was proposed to red uce signaling overhead using netw ork-based mobility manag emen $t$ without the need of host-based mobility stack at MN. However, PMIPv6 only supports intra-domain mobility and cannot support global mobility between domains. Figure 1 shows the architectures of MIPv6 and PMIPv6 [16]. MIPv6 supports mobility for the MN by providing it with at least two addresses: A fixe address called Home Address (HoA) is provided to Home Agent (HA). Care-of Address (CoA) is gained from foreign access netw ork and is chang ed when $\mathrm{MN}$ moves to a new subnet. Unlike MIPv6, PMIPv6 introduces two major elemen ts including Local Mobility Anchor (LMA) that manag es mobility -rela ted signaling of $\mathrm{MN}$ and Mobility Access Gatew ay (MAG). When $\mathrm{MN}$ hands over and chang es the access poin t from curren $t$ MAG to another MAG, the MN can use the same address it gained from the previous MAG. Theref ore, PMIPv6 provides the netw ork-based solution for processing MNą́rs localized mobility within Local Mobility Domain (LMD). PMIPv6 employs the per-MN-prefi model. Home Network Prefi (HMP), the unique code allocated to each MN, is not shared with other MNs. When MN moves within the PMIPv6 domain, the prefi follows MN and when MN moves within PMIPv6 domain except the firs access of MN in PMIPv6 domain, it does not require netw ork layer movemen $t$ detection or address configu ation processes. Thus the handov er latency and signaling overhead can be reduced significa tly. Also, beca use $\mathrm{MN}$ does not get involved in mobility -rela ted signaling in PMIPv6 environmen t, the two-way tunnel is gener ated between LMA and MAG instead of with $\mathrm{MN}$. As a resul $t$, this can assure the location privacy of $\mathrm{MN}$ [17]. 


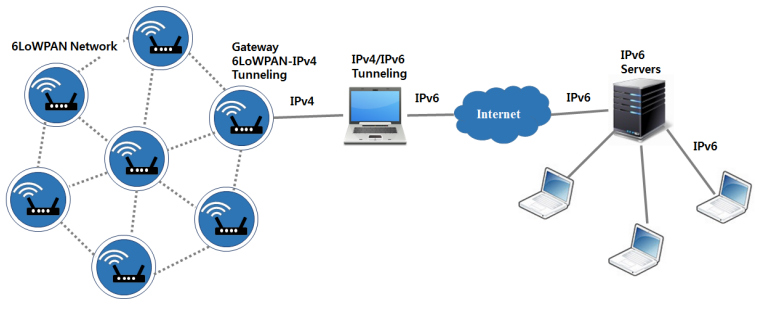

Figure 2. 6LoWPAN Network.

\subsection{GloWPAN}

The Internet Engineering Task Force (IETF) define IPv6 Low-pow er Personal Area Netw orks (6LoWPAN) which is an IPv6-based LoWPAN on the basis of IEEE 802.15.4 for comm unica tions with the Internet. 6LoWP AN (IPv6-based Low-pow er Wireless Personal Area Netw orks) is a IP sensor netw orking technol ogy to implemen $t$ a low power and low cost, theref ore, It is a technol ogy for a wireless environmen $t$ for IP-based applica tions. Conventional sensor netw ork technol ogy is less compatible with IP Netw orks. On the other hand, 6LoWP AN (IPv6-based Low-pow er Wireless Personal Area Netw orks ) which is one of the IP-USN technol ogy has an advantage that may be directly linked with the Internet infrastructure of the existing IPv4, IPv6, WiBro, WiFi, etc. With its vast address space, 6LoWPAN all ows global connectivity between a larg e number of IPv6 intellig ent devices over larg e areas. The protocol also enables the nodes to be self-organized i.e. can do self-detection, self-healing, and self-configuring without human intervention [18]. Figure 2 shows the architectures of 6LoWPAN Netw ork.

\section{Proposed Scheme}

\subsection{Operation procedures of sensor Proxy-AAA}

The adapta tion of authen tication in mobile IP handov er process can lead to excessiv e cost. Curren $t$ solutions cannot sufficiently meet these requiremen ts. To deal with these issues, this study proposes an advanced AAA authen tication scheme based on mobile IPv6. This proposed technique supports quick authen tication and introduces the concept of hier archical AAA to mobile IP combined with diameter protocol. In this proposed technique, AAA server will be implemen ted on Local Mobility Anchor (LMA) to implemen $\mathrm{t}$ simple and fast handover authen tication and hier archical authen tication as well as red uce intradomain authen tication cost. Proxy-AAA technique improves the previous authen tication schemes and binding upda ting methods in intra-domain handover and authen tication as well as inter-domain process [19]. In the process of intra-domain handover and authen tication, Proxy-AAA will reuse the session key based on LMA on HMIPv6. The proposed Proxy-AAA

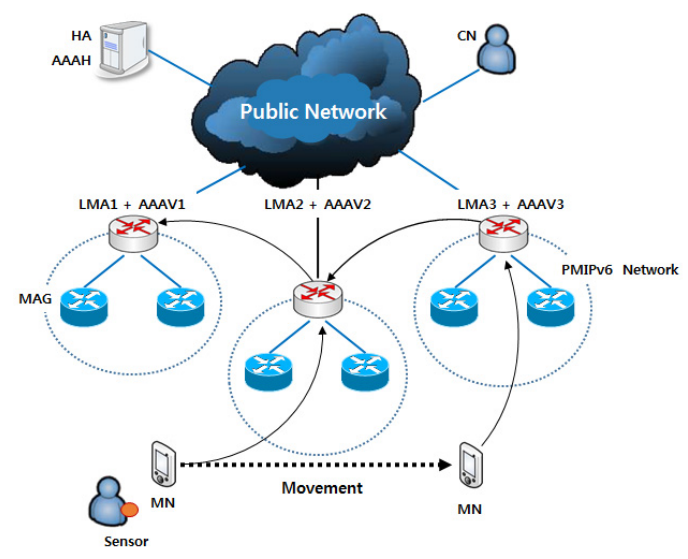

Figure 3. Forwarding scheme between different LMA.

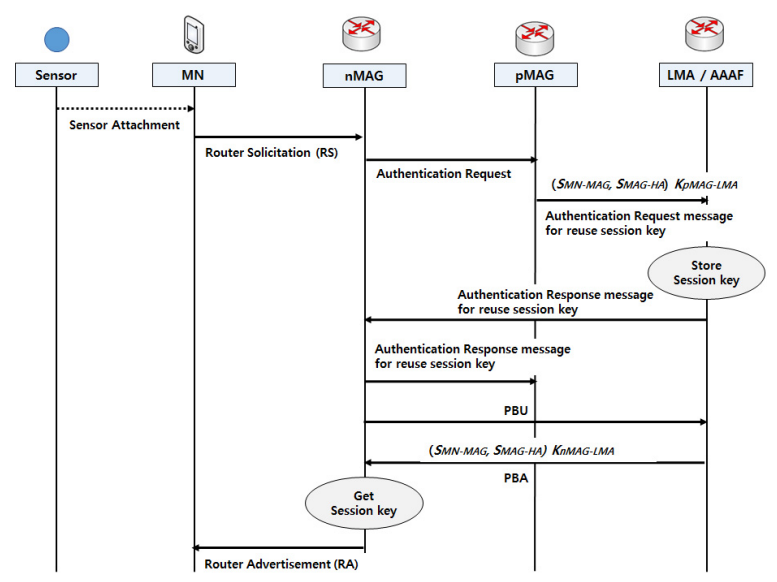

Figure 4. Intra-domain handover flow.

scheme adapts direct transmission strategy betw een LMAs in inter-domain handov er and authen tication process, and chooses the strategy for reusing session key on AAA server [20]. As shown in Figure 3, beca use information can be directl y deliv ered betw een LMAs in close vicinity, the control overhead of overall system can be saved compared to comm unica tion via HA.

When an MN moves into a netw ork region, from the left to the right in the figure it passes by LMA1, LMA2 and final y reaches LMA3. When an MN reaches LMA2, it immedia tely sends a BU messag e to LMA2. This will make LMA2 respond to LMA1. Upon receipt of the messag e, LMA1 compares the messag e with ones in the list of LMA, makes a request for informa tion on the MN, and upda tes the curren t LMA address of the MN. This will be followed by a direct transmission of packet data, from LMA1 to LMA2, without leveraging HA fail over.

Figure 4 shows the specifi $\mathrm{f}$ ow process of intradomain handover. Let's assume that data generated from sensor node within netw ork is being collected by adding the fixe data collection function called Resource Directory at MN. Also, beca use sensor node 


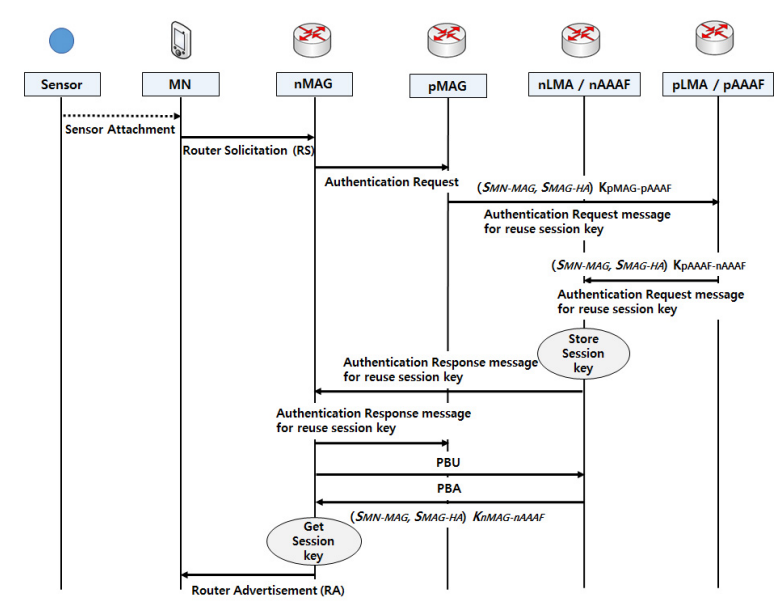

Figure 5. Inter-domain handover flow.

registers node name, type and lifetime in $\mathrm{RD}$, this $\mathrm{RD}$ can have the information of all nodes. MN deliv ers the collected data to information demander via internet working. On receiving notific tion from $\mathrm{MN}$, nMAG sends authen tication request messag e to pMAG to reuse the session key [21]. On receiving the request messag e, the mPAG encrypts session keys $S_{M N-M A G}$ and $S_{M A G-H A}$ using $K_{p M A G-L M A}$ and then delivers it to the LMA. LMA saves the receiv ed session key and returns the response messag e on reusing session key to nMAG. nMAG deliv ers the receiv ed response messag e to pMAG and sends PBU messag e to LMA. On receiving $\mathrm{PBU}$ messag e, LMA deliv ers $\mathrm{PBA}$ including the encrypted value of session keys $S_{M N-M A G}$ and $S_{M A G-H A}$ using $K_{n M A G-L M A}$ to nMAG. nMAG deliv ers the encrypted session key to $\mathrm{MN}$. Here, a reliable binding upda te channel between $\mathrm{MN}$ and LMA is formed. In addition, Figure 5 giv es the specifi messag e $\mathrm{f}$ ow in inter-domain handov er.

\subsection{Protocol selection}

To select the most appropria te mobility manag emen $\mathrm{t}$ protocol, the mobility manag ement protocol provided by netw ork and MN's mobility manag emen t protocol environmen $t$ need to be taken into consider ation. In the authen tication process, MAG searches MN's profil for MN's preference. From the search, in case MN's preferred protocol matches what was provided from access network, the matching protocol will be selected [22]. Otherwise, the MN's preference has higher priority. In case $\mathrm{MN}$ does not have a pref erence, the netw ork is responsible to assess the perf ormance of basic MIPv6 and Proxy-AAA technique and select the appropria te protocol. To evaluate the performance of basic MIPv6 and the Proxy-AAA scheme, the rela ted path latency is probed by MAG. While searching for path, MAG sends two types of proving messag es to
LMA several times. One is sent through nLMA and then redirected to PLMA and the related round-trip time (RTT) is denoted as $R T T_{\text {proxy-AAA }}$. The other probing messag e is sent directl y to pLMA and the rela ted RTT is denoted as $R T T_{\text {mip }}$. The average RTT of the MIPv6 path $\left(z_{n}\right)$ after path probing for times can be calcula ted as

$\overline{z_{n}}=\alpha R T T_{\text {mip }}(n)+(1-\alpha) \overline{z_{n-1}}$

Where $\alpha$ reflect the significanc of past events in the calcula tion of the weighted average. For exam ple, we set $\alpha$ to 0.8 in this paper, and then the most recen $t$ value $z_{n-1}$ will contribute to the calcula ted $z_{n}$ value with $20 \%$ weighting. This will avoid hysteresis if the value of $\alpha$ is carefull y selected [23]. The variable $z$ is initialized with the foll owing value:

$\overline{z_{0}}=R T T_{\text {mip }}(0)$

In a similar manner, the average RTT for the Proxy AAA scheme can be calculated and denoted as $t_{n}$. When path latency of MIPv6 hands over to much smaller and lower frequency than path latency and $\mathrm{MN}$ of Proxy-AAA technique, the performance of MIPv6 will be improved. On the other hand, in case the latency of MIPv6 is not much smaller than the latency of Proxy-AAA technique and hands over at higher frequency of $\mathrm{MN}$, the perf ormance of proposed ProxyAAA technique will be improved. In appropria tely selecting the better protocol according to netw ork condition and mobility parameters, protocol selection can be used.

$\frac{\overline{t_{n}}-\overline{z_{n}}}{N_{h}}<H_{t}, \quad$ select Proxy-AAA scheme

$\frac{\overline{t_{n}}-\overline{z_{n}}}{N_{h}} \geq H_{t}$, select Basic MIPv6

Here, $N_{h}$ is the handov er frequency and the value of $\overline{t_{n}}-\overline{z_{n}} / N_{h}$ is used as the quality indica tor to judge which protocol can provide better perf ormance, and $H_{t}$ is the quality threshol $\mathrm{d}$ to determine which protocol shoul d be selected.

\section{Performance Evaluation}

\subsection{System Modeling}

In this scheme, we construct an AAA server on the LMA residing in the visit domain (AAAV), and the AAA server is wholly responsible for accoun ting, authen tication, and authoriza tion of the MAG in the LMA domain of LMA. In the proxy-AAA method, the overhead of the entire system is composed of two parts: signaling control overhead $C_{\text {signal }}$ and 


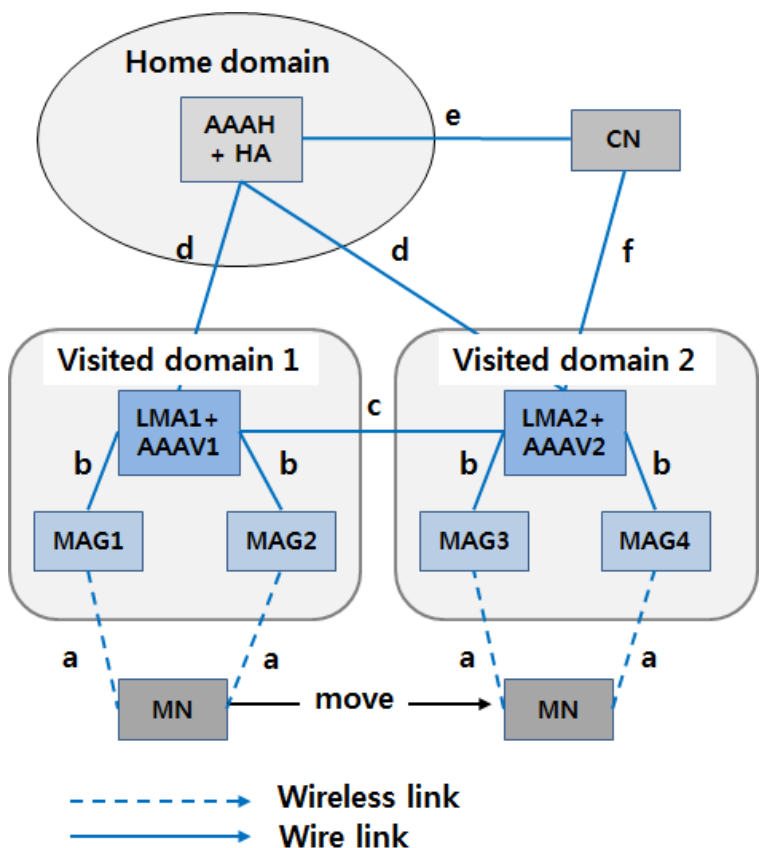

Figure 6. Cost analysis model of Proxy-AAA.

data transmission overhead $C_{\text {packet }}$. Signal control overhead is composed of authen tication signaling control overhead $C_{\text {auth }}$ and registr ation signaling control overhead $C_{r e g}$ in general, and $C_{r e g}$ is mainly made of the data transmission overhead from $\mathrm{CN}$ to $\mathrm{MN}\left(\mathrm{C}_{\mathrm{CN}-\mathrm{MN}}\right)$.

Figure 6 shows the netw ork topol ogy of a specifi Proxy-AAA for a system overhead analysis. As shown in Figure 6, the proposed hospital contains three foors, each with two wards. The hospital is considered as a one SPMIPv6 domain, in which sensor nodes are depl oyed on the patien t body as well as over the environmen $t$, ARs are used to control wards. Patien ts can get real- time care while moving between rooms, wards, and foors, or when the patient moves to another branch of the hospital.

$$
\begin{aligned}
C_{\text {total }} & =C_{\text {signal }}+C_{\text {packet }} \\
& =\beta\left(C_{\text {reg }}+C_{\text {auth }}\right)+\alpha C_{C N-M N}
\end{aligned}
$$

Here, $\alpha$ refers to the average velocity of packet data, transmitted from the $\mathrm{CN}$ to the $\mathrm{MN}$ (the average arriv al rate of packet data), and $\beta$ is the average switching rate of an $\mathrm{MN}$ when it transfers from a subnet to another, which is referred to as MN's switching rate per unit time [24]. When it is assumed that the number of packets transmitted from an $\mathrm{MN}$ to a $\mathrm{CN}$ remains constan $t$, we can express the packet to mobility ratio (PMR) of the packets receiv ed by the $\mathrm{MN}$ as $p=\alpha / \beta$. Also, $p=\alpha / \beta$ refers to the average number of packets receiv ed by a peer $\mathrm{CN}$. PMR is the ratio of packet arriv al rate and mobility rate, and it is a crucial indica tor for the presen $t$ study. The larger PMR is, the larger the arrival rate is than the mobility rate, meaning that the data transmission cost becomes larg er. When PMR becomes smaller, the arrival rate becomes smaller than the mobility rate, meaning the binding upda te cost becomes larger. Also, the average length of data packets is referred to as $l_{d}$, and signaling packets as $l_{s}$. The ratio of these is supposed to be $l=l_{d} / l_{s}$. For the convenience of calculation, $l_{d}=1024 \mathrm{~B}$ and $l_{s}=100 \mathrm{~B}$ are set by leveraging parameters offered by [25]. The overhead of transmitting signaling packets is associa ted with the distance betw een entities, while the overhead required for a data packet transmission should be $l$ times of that for a signaling packet transmission.

$$
\begin{aligned}
C_{\text {total }} & =\beta\left(C_{\text {reg }}+C_{\text {auth }}\right)+\alpha l_{d} L_{C N-M N} \\
& =\beta l_{s}\left(L_{\text {reg }}+L_{\text {auth }}\right)+\alpha l_{d} L_{C N-M N}
\end{aligned}
$$

The presen $t$ study adopts Ethernet LAN of $10 \mathrm{Mbit} / \mathrm{s}$ for the wired netw ork environmen $t$ and single-hop WLAN of $2 \mathrm{Mbit} / \mathrm{s}$ for the wireless environmen $\mathrm{t}$. For the calcula tion of time dela ys in wired and wireless links, we use an empirical formula respectiv ely expressed by $T_{r t}(h, k)$ and $W_{r t}(k)$ :

$T_{r t}(h, k)=3.63 k+3.21(h-1), W_{r t}(k)=17.1 k$

Where $k$ is for the packet length, with the unit $\mathrm{KB}$ (kilobytes), and $h$ is for the routing hops. The following section will provide some assum ptions. $\eta$ represen ts the cost of signaling packets in wired transmission per unit distance. The cost in wireless transmission is $10 \eta$. In addition, $\sigma$ represen ts the cost of data packets in wired transmission per unit distance. The cost in wireless transmission is $5 \sigma$.

$$
\begin{aligned}
C_{\text {packet }} & =\alpha C_{C N-M N} \\
& =\alpha l_{d}\left[\sigma\left(l_{C N-H A}+l_{H A-L M A}+l_{L M A-M A G}\right)\right. \\
& \left.+5 \sigma l_{M A G-M N}\right]
\end{aligned}
$$

MN's mobility is described by the simple equality $\mathrm{f}$ uid model. It is assumed that the area covered by the LMA is a $150 m \times 150 m$ square. When the pedestrian walks at a speed of 3 miles/hour (mph), $\beta=0.01$; when the vehicle travels at a speed of $60 \mathrm{mph}, \beta=0.2$. Then, 


$$
\begin{aligned}
C_{\text {packet }} & =\beta p l_{d}\left[\sigma\left(l_{C N-H A}+l_{H A-L M A}+l_{L M A-M A G}\right)\right. \\
& \left.+5 \sigma l_{M A G-M N}\right]
\end{aligned}
$$

As the suggested Proxy-AAA scheme aims to reduce the signaling overhead gener ated in authen tication and registr ation processes, this section compares ProxyAAA with traditional AAA schemes. Note that the traditional AAA is define as a simple combina tion of HMIPv6 and AAA. The relev ant parameters and definitio descriptions are shown in Table 1.

Table 1. The parameter Definition.

\begin{tabular}{l|l}
\hline Parameter & Definition \\
\hline$C_{M N-M A G}$ & $\begin{array}{l}\text { Signaling transmission cost between MN and } \\
\text { MAG }\end{array}$ \\
\hline$C_{M A G-L M A}$ & $\begin{array}{l}\text { Signaling transmission cost between MAG and } \\
\text { LMA }\end{array}$ \\
\hline$C_{H A-L M A}$ & $\begin{array}{l}\text { Signaling transmission cost between HA and } \\
\text { LMA }\end{array}$ \\
\hline$C_{L M A-L M A}$ & $\begin{array}{l}\text { Signaling transmission cost between LMA and } \\
\text { LMA }\end{array}$ \\
\hline$C_{A A A V-A A A H}$ & $\begin{array}{l}\text { Signaling transmission cost between AAAV } \\
\text { and AAAH }\end{array}$ \\
\hline$P_{M A G}$ & Signaling processing cost of MAG \\
\hline$P_{H A}$ & Signaling processing cost of HA \\
\hline$P_{L M A}$ & Signaling processing cost of LMA \\
\hline$P_{A A A}$ & Signaling processing cost of AAA \\
\hline
\end{tabular}

Assuming that MN moves out of the LMA region $m$ times in a certain period of time, then the authen tication will be perf ormed $m$ times. The earlier $m-1$ authen tications are intra-domain authen tications, and the last one is for inter-domain authen tication. Suppose that the authen tication process as a resul $t$ of MN's movement is in line with Poisson distribution with $\lambda$, then

$$
\begin{aligned}
p(n) & =\int_{t=0}^{\infty} p(n, t) f(t) d t=\int_{t=0}^{\infty} \frac{(\lambda t)^{n}}{n !} e^{-\lambda t} f(t) d t \\
& =\left.(-1)^{n} \frac{\lambda^{n}}{n !} \frac{d^{n} F(s)}{d s^{n}}\right|_{s=\lambda}
\end{aligned}
$$

Assuming that the time for which $\mathrm{MN}$ is in the region of LMA accords with Gamma distribution, and the expecta tion and variance of density function $f(t)$ can be expressed as $1 / \mu$ and $v$, the Laplace transf orm can be expressed as

$$
F(s)=(1+\mu v s)^{-1 / \mu^{2} v}
$$

$\frac{d^{n} F(s)}{d s^{n}}=(-\mu v)^{n}\left[\prod_{j=0}^{n-1}\left(\frac{1}{\mu^{2} v}+j\right)\right](1+\mu v s)^{-\left(\frac{1}{\mu^{2} v}+n\right)}$

However, $f(t)$ can vary depending on the exponen tial distribution, if $\mu^{2} v=1$. In that case, the expected authen tication time $(m)$ can be expressed as follows.

$E(m)=\sum_{n=1}^{\infty} n P(n)=\sum_{n=1}^{\infty} n \frac{\mu \lambda^{n}}{(\lambda+\mu)^{n+1}}=\frac{\lambda}{\mu}$

Through cost model analyses based upon HMIPv6, the inter-domain and intra-domain signaling overhead for a binding upda te under PMIPv6 can be expressed as foll ows.

$$
\begin{aligned}
B U_{\text {intra }}^{\text {PMIPv6 }} & =2 C_{M N-M A G}+2 C_{M A G-L M A} \\
& +2 P_{M A G}+P_{L M A} \\
B U_{\text {inter }}^{\text {PMIPv6 }} & =2 C_{M N-M A G}+2 C_{M A G-L M A}+2 C_{L M A-H A} \\
& +2 P_{M A G}+2 P_{L M A}+P_{H A}
\end{aligned}
$$

In addition, the authen tication delay under traditional AAA methods can be expressed as follows.

$$
\begin{aligned}
A^{\text {traditional }} & =2 C_{M N-M A G}+2 C_{M A G-L M A}+2 C_{L M A-A A A} \\
& +2 C_{A A A V-A A A H}+2 C_{L M A-H A}+P_{A A A} \\
& +2 P_{M A G}+4 P_{L M A}+P_{H A}
\end{aligned}
$$

Through this analysis, the entire signaling overhead in the LMA region under the traditional AAA scheme can be expressed as follows.

$$
\begin{aligned}
C_{\text {signal-traditional }} & =B U_{\text {total }}+A^{\text {traditional }} E(m) \\
& =B U_{\text {intra }}^{P M I P v 6}(E(m)-1) \\
& +B U_{\text {inter }}^{P M I P v 6}+A^{\text {traditional }} E(m)
\end{aligned}
$$

Through the analysis of the proposed Proxy-AAA scheme, the entire signaling overhead of its LMA region could be expressed as follows.

$$
\begin{aligned}
C_{\text {signal-proposed }} & =B U_{L M A}+A_{\text {intra }}^{\text {Proxy-AAA }}(E(m)-1) \\
& +A_{\text {inter }}^{\text {Proxy-AAA }} \\
& =B U_{\text {intra }}^{\text {PMIP } 6}(E(m)-1)+B U^{\text {Proxy-AAA }} \\
& +A_{\text {intra }}^{\text {Proxy-AAA }}(E(m)-1)+A_{\text {inter }}^{\text {Proxy-AAA }}
\end{aligned}
$$


It is assumed that under the proposed Proxy-AAA scheme, the binding upda te signaling cost incurred by MN movemen ts betw een LMA domains is expressed as follows.

$$
\begin{aligned}
B U^{\text {Proxy-AAA }} & =2 C_{M N-M A G}+2 C_{M A G-L M A} \\
& +2 C_{L M A-L M A}+2 P_{M A G}+3 P_{L M A}
\end{aligned}
$$

When using the Proxy-AAA scheme, LMA coexists with AAAV. Assuming $l_{L M A-A A A}=0$, then the authentication signaling overhead for inter and intra domain can be expressed as follows.

$$
\begin{aligned}
A_{\text {intra }}^{\text {Proxy-AAA }} & =2 C_{M N-M A G}+2 C_{M A G-M A G} \\
& +2 C_{M A G-L M A}+4 P_{M A G}+P_{L M A}
\end{aligned}
$$

$$
\begin{aligned}
A_{\text {inter }}^{\text {Proxy-AAA }} & =2 C_{M N-M A G}+2 C_{M A G-M A G}+2 C_{M A G-L M A} \\
& +C_{L M A-L M A}+2 C_{A A A V-A A A H}+4 P_{M A G} \\
& +2 P_{L M A}+P_{A A A}
\end{aligned}
$$

$$
\begin{aligned}
C_{M N-M A G} & =10 \eta l_{M N-M A G} l_{s} C_{M A G-M A G} \\
& =\eta l_{M A G-M A G} l_{s} C_{M A G-L M A} \\
& =\eta l_{M A G-L M A} l_{s}
\end{aligned}
$$

$$
\begin{aligned}
C_{L M A-H A} & =\eta l_{L M A-H A} l_{s} C_{L M A-L M A} \\
& =\eta l_{L M A-L M A} l_{S} C_{L M A-A A A} \\
& =\eta l_{L M A-A A A} l_{S}
\end{aligned}
$$

$C_{A A A V-A A A H}=\eta l_{A A A V-A A A H} l_{s}$

$$
\begin{aligned}
A_{\text {intra }}^{\text {Proxy }-A A A} & =\left(20 \eta l_{M N-M A G}+2 \eta l_{M A G-M A G}\right. \\
& \left.+2 \eta l_{M A G-L M A}\right) l_{s}+4 P_{M A G} \\
& +P_{L M A}
\end{aligned}
$$

$$
\begin{aligned}
A_{\text {inter }}^{\text {Proxy-AAA }} & =\left(\begin{array}{l}
20 \eta l_{M N-M A G}+2 \eta l_{M A G-M A G} \\
+2 \eta l_{M A G-L M A}+\eta l_{L M A-L M A} \\
+2 \eta l_{A A A V-A A A H}
\end{array}\right) l_{s} \\
& +4 P_{M A G}+2 P_{L M A}+P_{A A A}
\end{aligned}
$$

Assuming that the signaling overhead ratio of Proxy AAA and traditional AAA schemes is $R, R$ can be expressed as $R=C_{\text {signal-proposed }} / C_{\text {signal-traditional. }}$. The

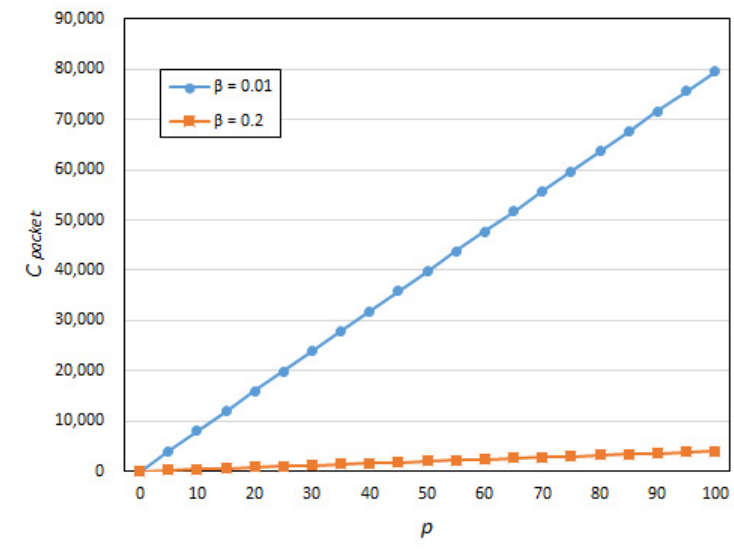

Figure 7. Packet data transmission overhead $(\mu=0.1)$.

average overhead for signaling can be expressed as $C_{\text {signal-a }}=C_{\text {signal-proposed }} / T_{a}$ by anal yzing the signaling overhead equation in the LMA region above. In this expression, $T_{a}$ refers to the average residence time in this LMA region. It shoul $\mathrm{d}$ be noted that the value must be smaller than 0.3 in the actual netw ork environmen $t$.

\subsection{Numerical Results}

This section will compare the system overhead. Specifi parameters and values are shown in Table 2.

Table 2. The parameter Definition.

\begin{tabular}{l|l|l|l}
\hline Parameter & Value & Parameter & Value \\
\hline$l_{M A G-L M A}$ & 5 & $l_{M N-M A G}$ & 1 \\
$P_{M A G}$ & 4 & $l_{H A-L M A}$ & 10 \\
$l_{L M A-L M A}$ & 10 & $l_{L M A-A A A}$ & 10 \\
$\sigma$ & 0.05 & $\eta$ & 0.1 \\
$P_{L M A}$ & 3 & $P_{H A}$ & 4 \\
$P_{A A A}$ & 3 & $l_{C N-H A}$ & 50 \\
$l_{M A G-M A G}$ & 1 & & \\
\hline
\end{tabular}

First of all, we establish MNs as a vehicle and pedestrian, analyzing their differen $t$ data pocket transmission overheads individ uall y. Figure 7 shows the data packet transmission overhead under a condition that MNs are pedestrians $(\beta=0.01)$ and vehicles $(\beta=$ 0.2 ). We can see that the data pocket transmission overhead $C_{\text {packet }}$ increases when the PMR $p$ increases.

Figure 8 shows how the data packet transmission overhead chang es when the value of PMR $p=10, p=50$ or $p=100$. We can see that as the average switching rate increases when the $\mathrm{MN}$ moves, the data packet transmission overhead $C_{\text {packet }}$ increases.

Figure 9 anal yzes the average signaling overhead of Proxy-AAA. This implies that the signaling overhead $C_{\text {signal }}$ increases with the increases as the arrival 


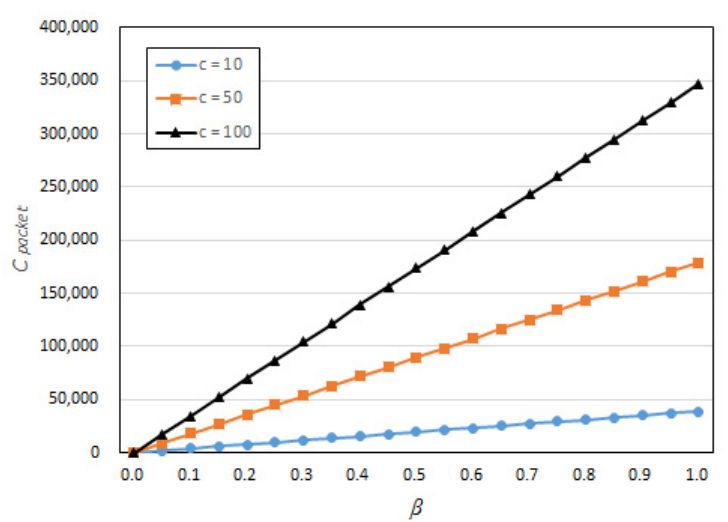

Figure 8. Packet data transmission overhead $(\mu=0.1)$.

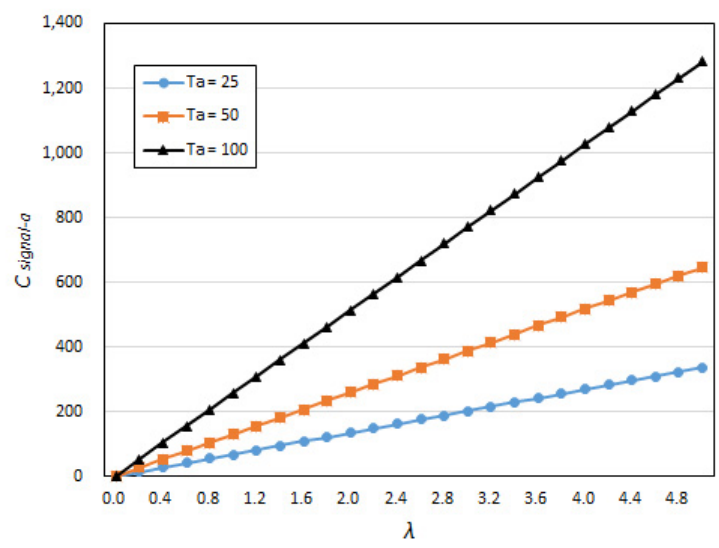

Figure 9. Signaling overhead $\left(l_{A A A V-A A A H}=50\right)$.

rate of authen tication events $\lambda$ increases. Frequen $t$ arriv al of $\mathrm{MN}$ brings increase in authen tica tion events arriv al rate and indica tes an increase in intra-domain authen tica tion in LMA domain and signaling overhead in registr ation.

Figure 10 analyzes the average signaling overhead of Proxy-AAA. This shows that the increase of residence time leads to a reduced signaling overhead $C_{\text {signal }}$.

Figure 11 analyzes the signaling overhead ratio $R$ betw een Proxy-AAA and traditional AAA schemes. This shows that $R$ must be less than 1 all times. In other words, the signaling overhead of the proposed Proxy AAA scheme is alw ays smaller than signaling overhead value of traditional AAA schemes irrelev ant of whether MN moves between domains or to the same LMA region.

Figure 12 shows the chang es of the signaling overhead ratio between traditional AAA schemes and Proxy-AAA schemes, when the value of $A_{A A A V-A A A H}=$

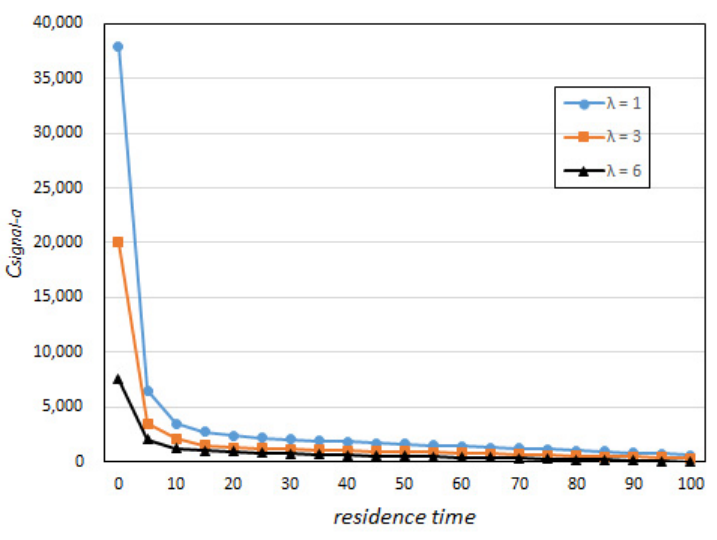

Figure 10. Signaling overhead $\left(l_{A A A V-A A A H}=50\right)$.

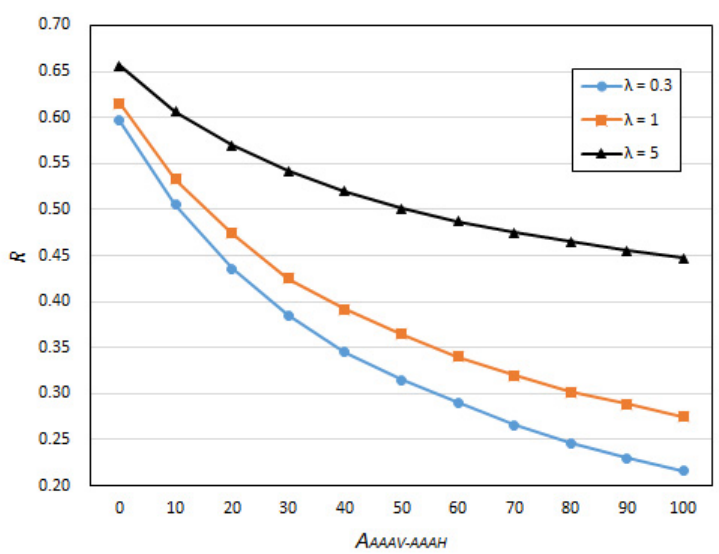

Figure 11. Ratio of signaling overhead (Proxy-AAA / traditional AAA).

10, $A_{A A A V-A A A H}=50$ and $A_{A A A V-A A A H}=100$, respectively. This shows that an increase in arrival rate of authen tication event reduces signaling overhead ratio betw een proposed Proxy-AAA schemes and traditional AAA schemes.

Figure 13 shows analysis of the entire overhead based on PMR $p$ increases $(\beta=0.01, \lambda=1)$. When the pedestrian $(\beta=0.01)$ moves, we can see that as the value of $p$ increases, entire overhead $C_{\text {total }}$ increases.

Figure 14 shows analysis of the entire overhead based on $\beta$ increases. When PMR fixed we can see that as the value of $\beta$ increases, entire overhead $C_{\text {total }}$ increases.

\section{Conclusions}

In this paper we proposed a sensor Proxy-AAA Authen tication Scheme based on Fast Handov er and Forwarding Mode for IP-based Internet of Things. In this study, the way of reducing the long dela y time and 


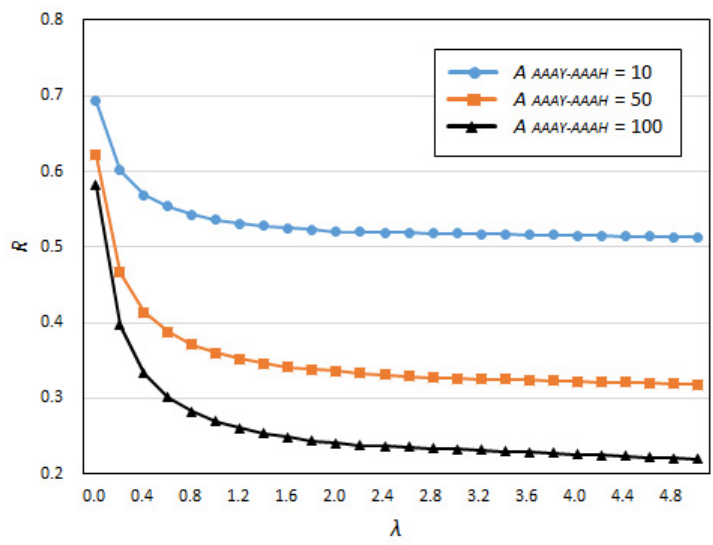

Figure 12. Change of signaling overhead ratio (Proxy-AAA / traditional $A A A)$.

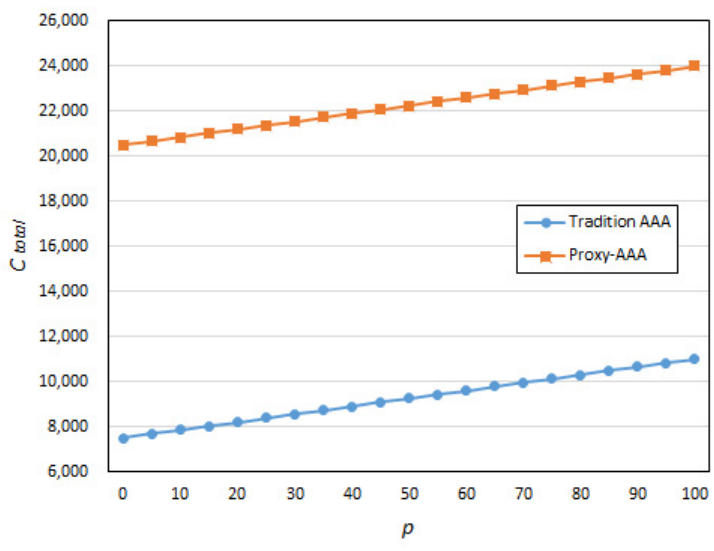

Figure 13. Figure 12 Total overhead $(\beta=0.01, \lambda=1)$.

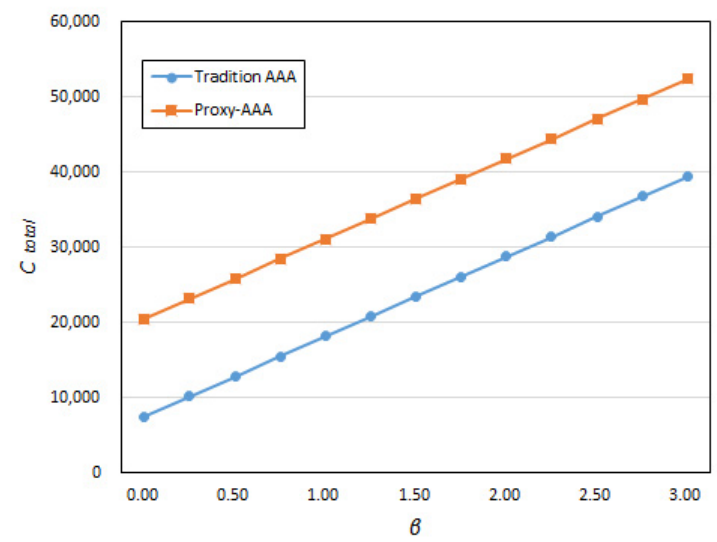

Figure 14. Figure 13 Total overhead $(p=30, \lambda=1)$. additional overhead from movemen $t$ of mobile device in mobile IP environmen $t$ by combining AAA and PMIPv6. This scheme has established a safe handov er by efficiently reducing signaling overhead generated by authen tication processes. Here we could confir that fast mobility mode and forwarding mode betw een various LMAs were supported. Moreover, the overall signaling overhead also show ed that proposed ProxyAAA scheme always has smaller value than previous traditional AAA schemes, hence enabling efficient movemen $t$ between domains by AAA Authen tication Scheme in forwarding mode at PMIPv6 supporting local mobility. Also, during movemen t betw een LMA domains, it was confirme that the farther the distance betw een RAAAS (Root AAA Server) and home domain, the higher the performance efficiency.

Acknowledgement. This work was supported by the Technol ogy Innovation Progr am (10054486, Developmen $\mathrm{t}$ of Open Industry IoT (IIoT) Smart Factory Platform and Factory -Thing Hardw are Technol ogy) funded By the Ministry of Trade, ind ustry \& Energy (MI, Korea).

This work was supported by ąřrThe Componen ts \& Materials Technol ogy Dev elopmen $t$ Progr am (10043800, Developmen $t$ of Micro Smart Environmen tal Sensor Measuremen t Module, Control Chip, and Applica tion Program)ąś funded by the Ministry of Trade, Ind ustry \& Energy (MI, Korea).

This work was supported by the Energy Technol ogy Developmen $t$ Progr am (2013T100200078, developmen $t$ of integrated demand response system technol ogy for coresiden tial resources and demonstr ation of business model) funded by the Ministry of Trade, Ind ustry \& Energy (MOTIE, Korea).

This work was supported by Global Creative Softw are (GCS) Project (No. 2014-044-028-001, Developmen t of ICT Convergence Technol ogy for Design Optimiza tion and Construction Technol ogy Innovation of the Structur al Frame and Envelope) funded by the Ministry of Science, ICT \& Future Planning (MSIP).

\section{References}

[1] C. de LaAt and G. Gross and L. Gommans and D. Spence (2000) Generic AAA Architecture(RFC 2903).

[2] Francesco Palmieri and Ugo Fiore and Aniello CAstiglione (2011) Automatic security assessment for next generation wireless mobile networks(Mobile Information Systems), Volume 7, pp. 217-239.

[3] P. Calhoun and J. Loughney and E. Guttman and G. Zorn and J. Arkкo (2003) Diameter Base Protocol(RFC 3588, September 2003).

[4] Le F and Patil B and Perkins C, et al (2004) Diameter mobile IPv6 application (Internet IETF Draft, 2004).

[5] Lee S Y and Hun E N and KIm S B, et AL (2005) An efficient performance enhancement scheme for fast mobility service in MIPv6(Proceedings of the Interna tional Conference on 
Computa tional Science and its Applica tions (ICCSA'05)), pp. 628-637, May 2005.

[6] KIM M and KIM M and Mun Y (2005) A hierarchical authentication scheme for MIPv6 node with local movement property(Proceedings of the Interna tional Conference on Computa tional Science and its Applica tions (ICCSA'05)), pp. 550-558, May 2005.

[7] Song Mei and WAng Li and Song Jun-De (2008) A secure fast handover scheme based on AAA protocol in mobile IPv6 networks(The Journal of China Universities of Posts and Telecomm unica tions, 15 (Sup1)), pp. 14-18, 2008.

[8] Ilsun You and Takahiro Hara (2010) Mobile and Wireless Networks(Mobile Information Systems), Volume 6, pp. 13, 2010.

[9] G. Giaretta (2009) Interactions between PMIPv6 and MIPv6: scenarios and related issues (draft-ietf-netlmm-mipinter actions-04, June 2009).

[10] Arjan Durresi and Mimoza Durresi and Leonard BAROLl (2008) Secure authentication in heterogeneous wireless networks(Mobile Informa tion Systems), Volume 4, pp. 119-130, 2008.

[11] Hakseon Hwang and Jongpil Jeong (2013) Reduction of Authentication Cost Based on Key Caching for InterMME Handover Support(The Journal of the Institute of Webcasting, Internet and Telecomm unication), Volume 13, pp.209-220, October 2013.

[12] Jeongbae Han and Seung-Hyun Lee and Dongryeol Shin and Jongpil Jeong (2011) Performance Analysis of Proxy-AAA Authentication Scheme in PMIPv6 Networks with Forwarding Mode Supporting(The Fourth Workshop on Information Technol ogies and Communication WOTIC 2011), pp. 81, October 2011.

[13] Seung-Hyun Lee and Dong-Ryeol Shin and Jongpil Jeong (2012) Performance Analysis of Proxy-AAA Authentication Scheme in PMIPv6 Networks with Forwarding Mode Supporting(Journal of Korean Society for Internet Information), Volume 13, pp.15-25, February 2012.

[14] Se-Won Yoo and Jongril Jeong (2012) Analytical Approach of Fast Inter-Domain Handover Scheme in Proxy Mobile IPv6 Networks with Multicasting Support(The KIPS Transactions:P artC), Volume 19C, pp.153-166, April 2012.

[15] Hyun-Suk Chai and Jongpil Jeong (2012) Security Analysis and Implementation of Fast Inter-LMA domain Handover Scheme in Proxy Mobile IPv6 Networks(The KIPS Transactions:P artC), Volume 19C, pp.99-118, June 2012.

[16] Jae-Hoon Kim and Jongpil Jeong (2011) Performance Analysis of Cost-Effective Handoff Scheme in PMIPv6
Networks with DNS Supporting(The 3rd International Conference on Internet - ICONI 2011), pp. 149-153, December 2011.

[17] J.-F. GuAn, Et AL (2009) Implementation and analysis of proxy MIPv6(Published Online, WCM), September 2009.

[18] A.J. Jara and M. A. Zamora and A. F. G. Skarmeta (2010) An Initial Approach to Support Mobility in Hospital Wireless Sensor Networks based on 6LoWPAN (HWSN6)(Journal of Wireless Mobile Networks, Ubiquitous Computing, and Dependable Applica tions), vol. 1, no. 2/3, pp. 107-122, 2010.

[19] SeungYoon Park and Jongpil Jeong (2013) On Pointer Forwarding Based Mobility Management for Cost-Optimized Proxy Mobile IPv6 Networks(2013 Seventh Interna tional Conference on Innov ative Mobile and Internet Services in Ubiquitous Computing - IMIS 2013), pp. 29-36, July 2013.

[20] Do-Kyoung Ra and Jongril Jeong (2012) Cost-Effective Mobility Management Scheme in Proxy Mobile IPv6 Networks with Function Distributor Support(The Journal of the Institute of Webcasting, Internet and Telecomm unication), Volume 12, pp.97-107, February 2012.

[21] J. Jeong and M. KANG and Y. Chо and J. Сног (2013) 3S: Scalable, secure and seamless inter-domain mobility management scheme in proxy mobile IPv6 networks(Interna tional Journal of Security and its Applica tions), Volume 7, Issue 4, pp. 51-70, July 2013.

[22] Jongyoun Kim and Jongsun Park and Jongpil Jeong (2012) LC-GM2: Low-Cost Global Mobility Management Scheme in Proxy Mobile IPv6 Networks(KIPS Transactions on Computer and Communica tion Systems), Volume 1, pp.193-204, December 2012.

[23] D.-G. Anderson (2005) Improving end-to-end availability using overlay networks(Massachusetts Institute of Technology), February 2005.

[24] Jain R and Raleigh T and Graff C, et al (1998) Mobile Internet access and QoS guarantees using mobile IP and RSVP with location registers(Proceedings of Interna tional Conference on Communications (ICC'98)), Vol 3, pp. 1690-1695, June 1998.

[25] LeE K and Mun Y (2005) An efficient macro mobility scheme supporting fast handover in hierarchical mobile IPv6(Proceedings of the International Conference on Computa tional Science and its Applica tions (ICCSA'05)), pp. 408-417,Ma y 2005. 